\title{
Geographical Information Systems: A Tool to Map and Analyze Disease Spread
}

\author{
Indumathi Srinath ${ }^{\star 1}$, Barbara Szonyi ${ }^{1}$, Maria Esteve-Gassent ${ }^{1}$, Blanca Lupiani ${ }^{1}$, Raju \\ Gautam¹, Alfonso Clavijo², Sang-shin Park¹ and Renata Ivanek-Miojevic ${ }^{1}$
}

${ }^{1}$ Texas A \&M, College station, TX, USA; ${ }^{2}$ Texas Veterinary Medical Diagnostic Laboratories, College station, TX, USA

\section{Objective}

Use GIS to illustrate and understand the association between environmental factors and spread of infectious diseases.

\section{Introduction}

Spatial methods are an important component of epidemiological research motivated by a strong correlation between disease spread and ecological factors (1). Our case studies examine the relationship between environmental conditions, such as climate and location, and vector distribution and abundance. Therefore, GIS can be used as a platform for integrating local environmental and meteorological variables into the analysis of disease spread, which would help in surveillance and decision making.

\section{Methods}

Case study 1- Lyme disease -Lyme disease is a tickborne infection caused by the bacterium Borrelia burgdorferi. The goal of this study was to analyze the association between meteorological factors and Lyme disease risk in humans in Texas. A total of 1,212 cases reported from 138 counties in Texas from Jan 2000 to Dec 2010 were analyzed. We used temperature and precipitation raster grids to generate humidity maps for Texas region. Our results indicated that there is a strong positive association between Lyme disease incidence and humidity, with western cross timbers region having a higher risk then the low plains.

Case study 2- Spinach - Motivated by the recent increase in foodborne outbreaks related to fresh produce, one of the objectives for this study was to use the geospatial analysis to elucidate factors that contribute to contamination of produce at preharvest. We collected 955 spinach samples from 13 produce farms in Colorado and Texas during 2010-2011 and tested the samples for Listeria monocytogenes, Escherichia coli O157:H7 and Salmonella contamination. The spinach contamination results were then used in conjunction with the National Resource Information (NRI) databases along with the SSURGO database to predict environmental and meteorological factors contributing to spinach contamination. Our findings would help to reduce frequency of human foodborne illnesses related to fresh produce.

Case study 3- Valley fever Coccidioidomycosis or Valley Fever (VF) is a fungal zoonosis affecting humans and a variety of animal species. In this study, we used Texas Veterinary Medical Diagnostic Laboratories (TVMDL) data of all dog sera tested for coccid- ioidomycosis from July 1999 - December 2009. Census data on human population density for Texas were used to determine the dog population density and identify disease clusters for the 5,871 submitted dog sera over a period of 10.5 years. Both the isopleth map of the VF seropositive rates in dogs across Texas and the identified spatial and spatio-temporal clusters of the disease suggested that VF occurs in the western and southwestern part of Texas at a much higher rate than in other areas of the state (2). Since VF is not a reportable disease in TX, dogs could be used as a sentinel for human infection.

\section{Results}

The above studies illustrate the utility of GIS as a tool in integrating different ecological factors to understand disease occurrence and spread. The geographical and temporal patterns found in these studies provide benchmark to support disease control activities in Texas. Additionally, the identification of high-risk areas may be useful for decision makers to improve and prevent future disease spread.

\section{Conclusions}

Spatial epidemiological research has challenges, such as dealing with coarse level and aspatial datasets. Testing laboratories provide limited spatial information up to the zip code level due to the confidentiality concerns. Spatial analysis of such dataset prevents research at finer resolutions (Census block or block group). Despite these limitations, spatial epidemiology continues to be an invaluable field in the research and surveillance of infectious disease.

\section{Keywords}

Geospatial analysis; Disease mapping; Environmental variables

\section{References}

1. Auchincloss AH, Gebreab SY, Mair C and Diez Roux AV. A Review of Spatial Methods in Epidemiology, 2000-2010. Annu. Rev. Public Health. 2012; 33:107-22.

2. Gautam R, Srinath I, Clavijo A, Szonyi B, Bani-Yaghoub B, et al. Identifying Areas of High Risk of Human Exposure to Coccidioidomycosis in Texas Using Serology Data from Dogs. Zoonoses and Public Health. Article first published online: 1Aug,2012

*Indumathi Srinath

E-mail: isrinath@webmail.cvm.tamu.edu 